$\mathbb{T}$ periodica polytechnica

Mechanical Engineering

51/2 (2007) 83-87

doi: 10.3311/pp.me.2007-2.07

web: http://www.pp.bme.hu/me

(c) Periodica Polytechnica 2007

RESEARCH ARTICLE

\section{Dynamical investigation of a linear motor to be used in cellular phones}

Attila Lukács / Zsolt Szabó

Received 2008-01-18

\begin{abstract}
Nowadays vibrating alert is a common feature of cellular phones. For this purpose a radial flux linear-motor with airgap solenoid was developed, which operates in the vicinity of its natural frequency. This paper presents the amplification diagram of the mechanical model of this new construction obtained both numerically and analytically. Furthermore, the results are compared to measurement data which are showing nonlinear dynamical behaviour.
\end{abstract}

\section{Keywords}

electromagnetic actuator · vibrating alert $\cdot$ forced Duffing equation

\section{Acknowledgement}

This research was partially supported by the Hungarian Science Foundation OTKA Grant No. F47318. The authors also wish to thank Prof. Gábor Stépán for his valuable comments and dedicate this paper to him on the occasion of his election as full member of the Hungarian Academy of Science.

\section{Attila Lukács}

Department of Mechatronics, Optics and Instrumentation Technology, BME, H1111 Budapest, Múegyetem rkp. 5., Hungary

e-mail: lukacs@mogi.bme.hu,

\section{Zsolt Szabó}

Department of Applied Mechanics, BME, H-1111 Budapest, Múegyetem rkp. 5., Hungary

e-mail: szazs@mm.bme.hu

\section{Introduction}

Electromagnetic actuators rotate shafts, force masses for periodic translational motion. They can be used for both interrupted and stationary working. The actuator itself is an electromechanical (or mechatronical) system in which the moving mechanical part has a feed-back on the operating electronic circuit $[4,6]$. In the simplest case, such an actuator has one mechanical and one electrical degree of freedom [1]: the position of the moving mass and the electric current in the circuit. However, the equation of motion of the mass is a second order differential equation due to Newton's Second Law while Kirchhoff's Voltage Law yields a first order differential equation at the most.

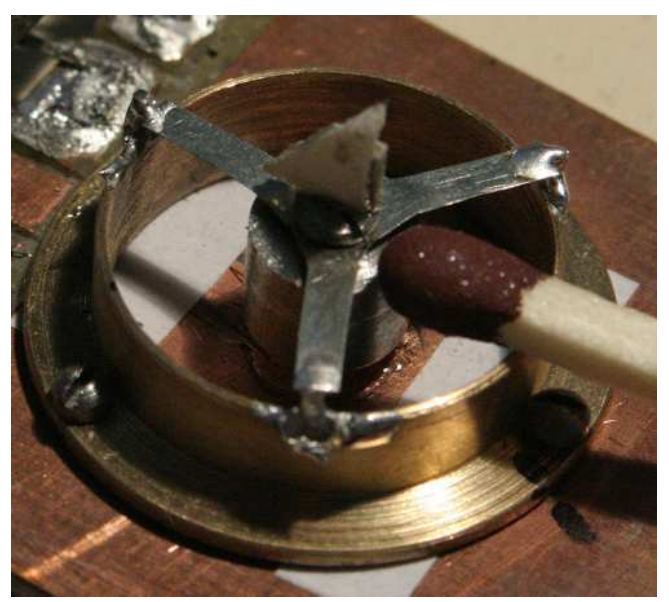

Fig. 1. Photo of the investigated electro-magnetic actuator

Fig. 1 shows the mechatronical construction in question. Its size is in the mm-range. The main application field is to create mechanical excitation in cellular phones $[2,5,7]$. The actuator in the picture was made by the authors as the prototype of a radial flux linear motor with air-gap solenoid.

\section{System model of the electro-magnetic actuator}

In Fig. 2 an exploded sketch of the actuator can be seen. The permanent magnet $(\mathrm{NdFeB})$, the bell-shaped flux-closing part and the ring-shaped air-gap build the magnetic circuit which is, at the same time, the moving part of the actuator. Fixing it on a flexible suspension yields a one-degree-of-freedom oscillator 
of which mass $m$ is only 1 gramm. The electric circuit contains the solenoid coil and the excitation voltage source $U(t)$. The coil has $n$ turns of length $l$. The electro-mechanical model is shown in Fig. 3. The resistor $R$ and the inductor $L$ take into consideration the resistance and the self-inductance of the coil.

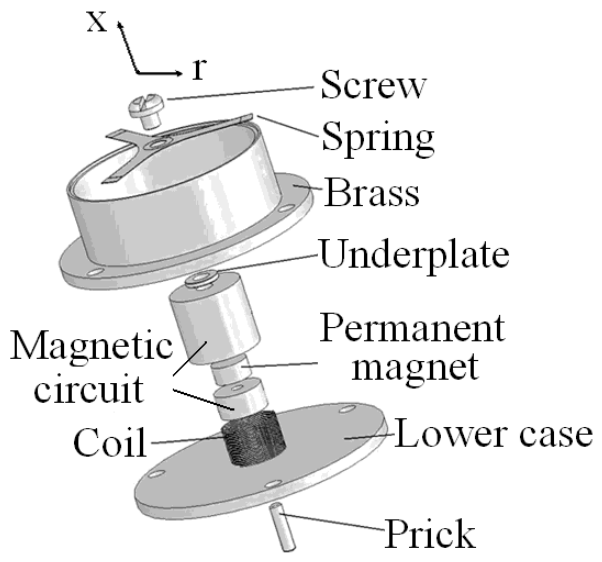

Fig. 2. Exploded sketch of the actuator

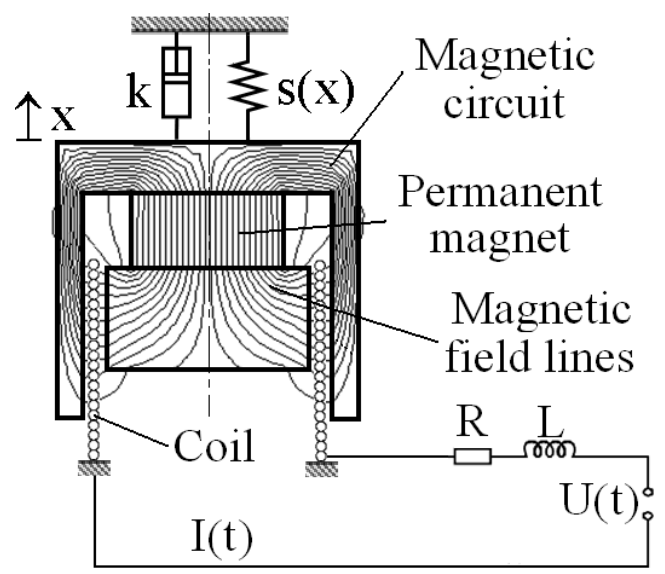

Fig. 3. Mechatronical system model

This system has three state variables: position $x(t)$ and velocity $\dot{x}(t)$ of the mass $m$, and current $I(t)$ of the electric circuit. The governing equations according to Newton's Second Law and Kirchhoff's Voltage Law are as follows:

$$
\begin{aligned}
m \ddot{x} & =-F_{s}(x)-k \dot{x}+F_{B}(I), \\
L \dot{I}+U_{B}(\dot{x})+R I & =U(t),
\end{aligned}
$$

where $F_{S}(x)$ is the nonlinear spring force, $k$ is the damping coefficient of the mechanical part, and $F_{B}(I)$ is the Lorentz force reacting on the magnet from the coil containing current $I$ in a magnetic field of induction $B$ :

$$
F_{B}(I)=-I\left(n l \mathbf{e}_{t}\right) \times\left(B \mathbf{e}_{r}\right) \cdot \mathbf{e}_{x} \equiv \operatorname{Inl} B, \quad\left(\mathbf{e}_{r} \mathbf{e}_{t} \mathbf{e}_{x}=1\right)
$$

because the current in the turns of the coil is perpendicular to the radial magnetic field in the air-gap $\left(\mathbf{e}_{r}, \mathbf{e}_{t}\right.$ and $\mathbf{e}_{x}$ denote the radial, tangential and axial unit vectors, respectively). The formula $U_{B}(\dot{x})$ represents the counter electromotive force which is induced by the moving permanent magnet:

$$
U_{B}(\dot{x})=\dot{x} n l B .
$$

The spring force was assumed to have cubic nonlinearity: $F_{s}(x)=s\left(x+\mu x^{3}\right)$, because of the small dimensions of the suspension spring (its thickness is only $50 \mu \mathrm{m}$ ) and its relatively "large" deformation (it can be even $0.25 \mathrm{~mm}$ ). The linear spring stiffness $s$ and the coefficient $\mu$ of the nonlinear term was determined both by finite element analysis using ANSYS and experimentally. The results are in good agreement, as it is shown in Fig. 4. The function fitted on the experimental data is as follows:

$$
F_{S}(x)=0.51\left[\frac{\mathrm{N}}{\mathrm{mm}}\right]\left(x+26\left[\mathrm{~mm}^{-2}\right] x^{3}\right) .
$$

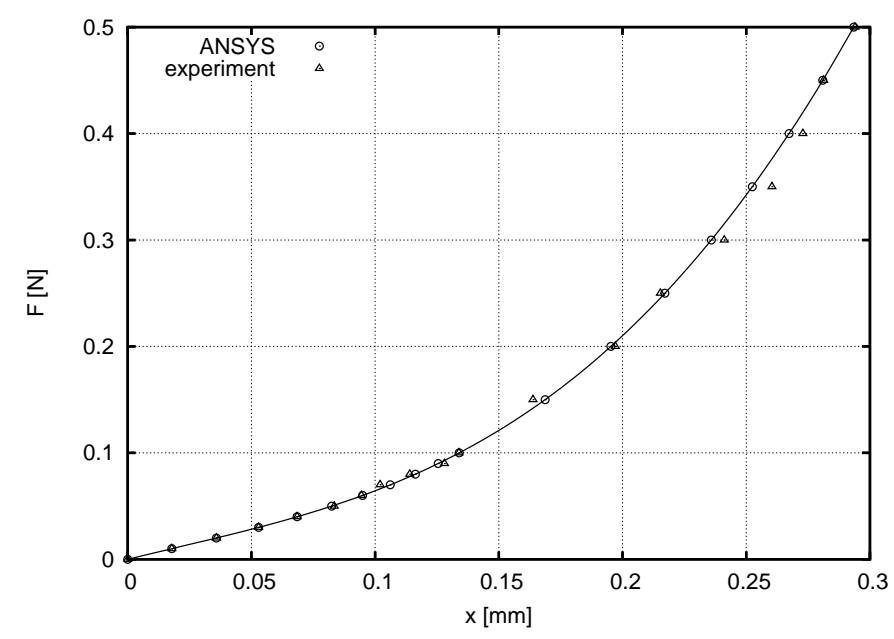

Fig. 4. Nonlinear characteristic of the spring force

Substituting formulas (3), (5) and (4) into Eq. (1) and Eq. (2) respectively, we obtain the following differential equations:

$$
\begin{aligned}
\ddot{x}+2 D \alpha \dot{x}+\alpha^{2}\left(x+\mu x^{3}\right) & =\frac{1}{m} B \ln I, \\
L \dot{I}+B \ln \dot{x}+R I & =U_{0} \cos \omega t,
\end{aligned}
$$

where $\alpha^{2}=s / m, 2 D \alpha=k / m$ and $U_{0} \cos \omega t$ is the sinusoidal excitation voltage of a low-frequency generator. The experimentally determined values of system parameters are summarized in Table 1.

Because of its small value, the inductance can be neglected. This assumption was confirmed by numerical simulations, i.e. it is satisfactory considering only the following second order differential equation:

$$
\ddot{x}+\left(2 D \alpha+\frac{(B \ln )^{2}}{m R}\right) \dot{x}+\alpha^{2}\left(x+\mu x^{3}\right)=\frac{B \ln }{m R} U_{0} \cos \omega t .
$$

Fig. 5 shows the obtained amplification diagram $A(\omega)$ of system (8) and the relative error comparing to the simulation results $A_{L}(\omega)$ where the inductance $L$ was taken into account:

$$
\varepsilon(\omega)=\frac{\left|A(\omega)-A_{L}(\omega)\right|}{A_{L}(\omega)} .
$$


Tab. 1. List of system parameters

\begin{tabular}{ll}
\hline System parameter & \multicolumn{1}{c}{ Value } \\
\hline mass: & $m=0.001 \mathrm{~kg}$ \\
relative damping: & $D=0.065$ \\
angular eigenfrequency: & $\alpha=714 \mathrm{rad} / \mathrm{s}=114 \mathrm{~Hz}$ \\
nonlinear spring coefficient: & $\mu=26 \times 10^{6} 1 / \mathrm{m}^{2}$ \\
number of turns: & $n=30$ \\
length of a turn: & $l=0.014 \mathrm{~m}$ \\
magnetic induction ${ }^{\star}:$ & $B=0.22 \mathrm{~T}$ \\
resistance: & $R=10 \Omega$ \\
inductance: & $L=0.053 \mathrm{mH}$ \\
amplitude of the excitation voltage: & $U_{0}=2.46 \mathrm{~V}$ \\
\hline${ }^{\star}$ : obtained numerically using ANSYS)
\end{tabular}

It can be seen that the error is small relative to the vibration amplitude, thus it can be neglected, indeed.

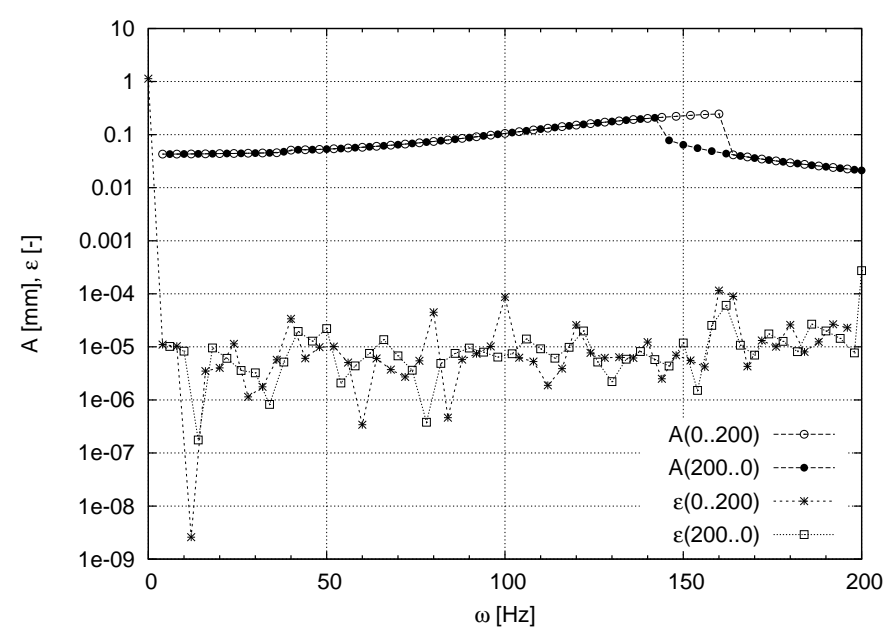

Fig. 5. Simulated amplification diagram $(A[\mathrm{~mm}])$ and its relative error $(\varepsilon)$ w.r.t. the original system $(0 \rightarrow 200$ : for increasing frequencies, $200 \rightarrow 0$ : for decreasing frequencies)

\section{Analytical investigations}

Eq. (8) is a forced damped Duffing equation with cubic nonlinearity. For the sake of simplicity, we neglect the effect of damping:

$$
\ddot{x}+\alpha^{2} x\left(1+\mu x^{2}\right)=\alpha^{2} f_{0} \cos \omega t,
$$

where

$$
f_{0}=\frac{B \ln }{\alpha^{2} m R} U_{0} \approx 0.045[\mathrm{~mm}]
$$

\subsection{Autonomous case}

If there is no excitation ( $\left.f_{0}=0\right)$, according to Poincaré's method of small parameters [3] the solution can be assumed as

$$
x(t)=x_{0} \cos (\gamma t+\delta)+\mu x_{1}(t)+O\left(\mu^{2}\right),
$$

where

$$
\gamma=\alpha+\mu h_{1}+O\left(\mu^{2}\right)
$$

is the modified angular eigenfrequency and $h_{1}$ is an unknown parameter, which can be determined as follows.
Let us consider only the solution belonging to the following initial conditions:

$$
x(0)=x_{0}, \quad \dot{x}(0)=0, \quad\left(x_{1}(0)=0, \dot{x}_{1}(0)=0, \cdots\right)
$$

which implies $\delta=0$.

Now, Eq. (9) can be rewritten as

$$
\ddot{x}+\left(\gamma-\mu h_{1}\right)^{2} x\left(1+\mu x^{2}\right) \approx 0,
$$

and if we put (10) into (13) and separate the coefficients of $\mu$ it yields:

$$
\begin{array}{ll}
\mu^{0}: & \left(-\gamma^{2}+\gamma^{2}\right) x_{0} \cos \gamma t=0, \\
\mu^{1}: & \ddot{x}_{1}(t)+\gamma^{2} x_{1}(t)=2 h_{1} \gamma x_{0} \cos \gamma t-\gamma^{2} x_{0}^{3} \cos ^{3} \gamma t, \\
\mu^{2}: & \cdots
\end{array}
$$

Since

$$
\cos ^{3} \gamma t=\frac{1}{4} \cos 3 \gamma t+\frac{3}{4} \cos \gamma t
$$

and the resonant solution should be avoided, the coefficient of $\cos \gamma t$ has to be zero in the equation belonging to $\mu^{1}$, i.e.

$$
2 h_{1} \gamma x_{0}-\frac{3}{4} \gamma^{2} x_{0}^{3}=0 \Rightarrow h_{1}=\frac{3}{8} \gamma x_{0}^{2} .
$$

Thus, the base frequency can be obtained from formulas (11) and (14):

$$
\gamma \approx \frac{\alpha}{1-\frac{3}{8} \mu x_{0}^{2}}=\frac{114}{1-\frac{3}{8} 26 \cdot 0.1^{2}} \equiv 126[\mathrm{~Hz}] .
$$

The solution that satisfies the initial conditions (12) for $x_{1}(t)$ is

$$
x_{1}(t)=\frac{x_{0}^{3}}{32}(\cos 3 \gamma t-\cos \gamma t),
$$

hence,

$$
x(t)=\left(x_{0}-\mu \frac{x_{0}^{3}}{32}\right) \cos \gamma t+\mu \frac{x_{0}^{3}}{32} \cos 3 \gamma t+O\left(\mu^{2}\right) .
$$

\subsection{Nonautonomous case}

Let us rewrite Eq. (9) into dimensionless form:

$$
x^{\prime \prime}+x\left(1+\mu x^{2}\right)=f_{0} \cos \lambda \tau,
$$

where $\tau=\alpha t$ is the dimensionless time, ' denotes $d / d \tau$ and $\lambda=\omega / \alpha$ is the frequency ratio.

The particular solution of the linearized system $(\mu=0)$ :

$$
x(\tau)=\frac{f_{0}}{1-\lambda^{2}} \cos \lambda \tau
$$

After some trigonometric considerations (e.g. $\cos ^{3} \lambda \tau$ causes terms of $\cos 3 \lambda \tau$ to appear), we assume the stationary solution of the nonlinear system as follows:

$$
x(t)=x_{0}(\mu) \cos \lambda \tau+\mu x_{1}(\mu) \cos 3 \lambda \tau+O\left(\mu^{2}\right),
$$

which means that the higher frequency terms have less and less significance in $x(t)$. 
After putting (18) into Eq. (16) and separating the coefficients of $\cos \lambda \tau, \cos 3 \lambda \tau$, etc. we get:

$$
\begin{aligned}
\cos \lambda \tau: & \left(-\lambda^{2}+1\right) x_{0}+\mu \frac{3}{4} x_{0}^{3}=f_{0} \\
\cos 3 \lambda \tau: & \left(-9 \lambda^{2}+1\right) x_{1}+\mu \frac{1}{4} x_{0}^{3}=0 \\
\cos 5 \lambda \tau: & \cdots
\end{aligned}
$$

The equation for the coefficients of $\cos \lambda \tau$ yields the nonlinear amplification diagram in implicit form:

$$
\left(1-\lambda^{2}\right) x_{0}+\frac{3}{4} \mu x_{0}^{3}-f_{0}=0,
$$

from which one can obtain the solution for the linear case $(\mu=$ 0 ) as shown in Eq. (17).

The result of (19) can also be obtained by rewriting Eq. (16) in the following form:

$$
x^{\prime \prime}+\lambda^{2} x=\mu\left(\Psi x-x^{3}+\Phi \cos \lambda \tau\right),
$$

where

$$
\Psi=\frac{\lambda^{2}-1}{\mu}, \quad \Phi=\frac{f_{0}}{\mu}
$$

and applying Poincaré's method $\left(x(\tau)=x_{0}(\tau)+\mu x_{1}(\tau)+\cdots\right.$, etc.).

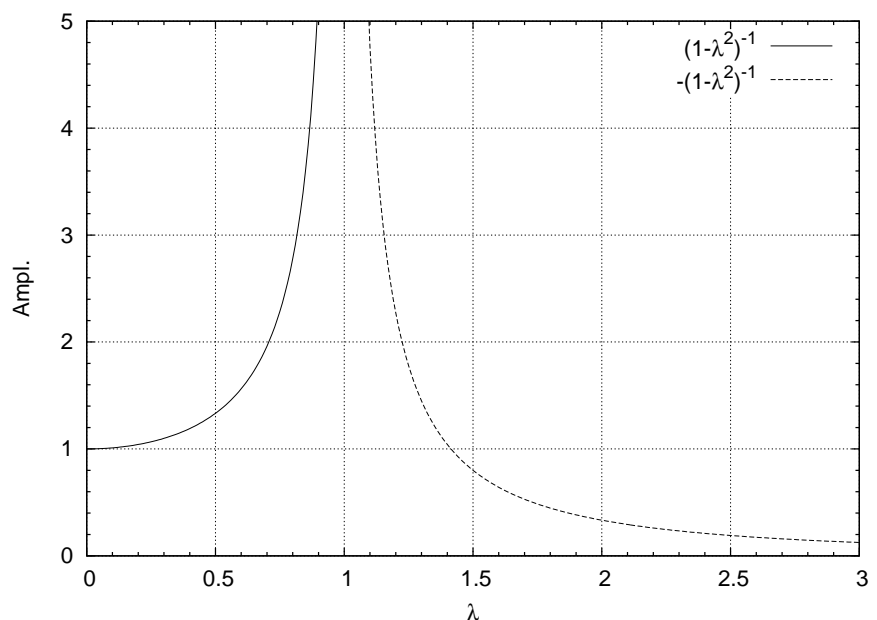

Fig. 6. Dimensionless amplification diagram for the linear case $(\mu=0)$

\subsection{The nonlinear amplification diagram}

The graph of the resonance curve of the linear system is not a continuous function and usually its magnitude is plotted (see Fig. 6):

$$
\left|x_{0}\right|=\frac{f_{0}}{\left|1-\lambda^{2}\right|} \equiv \frac{1}{1-\lambda^{2}} \begin{cases}+f_{0}, & \text { if } \lambda<1 \text { (left or upper branch) } \\ -f_{0}, & \text { if } \lambda>1 \text { (right or lower branch) }\end{cases}
$$

Hence, for nonlinear case $(\mu>0)$ the deformation of these branches is described by the modified forms of Eq. (19), which are also shown in Fig. 7:

$$
\begin{aligned}
\left(1-\lambda^{2}\right) x_{0}+\frac{3}{4} \mu x_{0}^{3} & =+f_{0}, \text { (upper branch) } \\
\left(1-\lambda^{2}\right) x_{0}+\frac{3}{4} \mu x_{0}^{3} & =-f_{0}, \text { (lower branch). }
\end{aligned}
$$



Fig. 7. Nonlinear amplification diagram $\left(f_{0}=0.045 \mathrm{~mm}\right)$

In the limit case $f_{0} \rightarrow 0$ the two branches huddle up against the hyperbola of the so called skeleton curve:

$$
\lambda^{2}-\frac{x_{0}^{2}}{\frac{4}{3 \mu}}=1 .
$$

One can show that the greater amplitudes $\left(x_{0}>x_{0}^{\star}\right)$ of the lower branch belong to unstable solutions and experimentally cannot be detected. It is also interesting to determine the limit frequency $\lambda^{\star}$ below which only one solution exists, which is stable. This can be achieved by derivating Eq. (22) (lower branch) with respect to $x_{0}$ and substituting $\lambda^{\prime}\left(x_{0}^{\star}\right)=0$ into it:

$$
1-\left(\lambda^{\star}\right)^{2}+\frac{9}{4} \mu\left(x_{0}^{\star}\right)^{2}=0 .
$$

From Eqs. (22) and (23) we obtain:

$$
\begin{aligned}
x_{0}^{\star} & =\sqrt[3]{\frac{2 f_{0}}{3 \mu}} \equiv \sqrt[3]{\frac{0.09}{78}} \approx 0.105[\mathrm{~mm}], \\
\lambda^{\star} & =\sqrt{1+\sqrt[3]{\frac{81}{16} \mu f_{0}^{2}}} \approx 1.28 .
\end{aligned}
$$

For our physical system this means:

$$
\omega^{\star}=\alpha \sqrt{1+\sqrt[3]{\frac{81}{16} \mu\left(\frac{B \ln U_{0}}{s R}\right)^{2}}} \approx 146[\mathrm{~Hz}] .
$$

\section{Experimental results}

As the analytical investigations have previously showed, the amplitude of the forced vibration depends on the frequency of the harmonic excitation in the linear and nonlinear case, as well. The perfectness of our mechatronical system model described by Eq. (8) was tested by comparing the vibration curves obtained from numerical simulation and experiments, respectively. It is very important to determine the correct mathematical model (governing equations) and its dynamical parameters in order to design the electronics of the built actuator. 
During the experiments, the a displacement amplitude was measured by a transmission opto-gate (TIL 138). Such optoelectronical transducers are typically used in measurements where the displacements are in the submillimeter range. Their main advantage is that they do not influence the object to be measured and they have relatively high cut-off frequency. The results of the experiment and the simulation can be seen in Fig. 8 which shows good agreement. In Fig. 9 the difference from the undamped analytical results can be seen, which is also not too large.

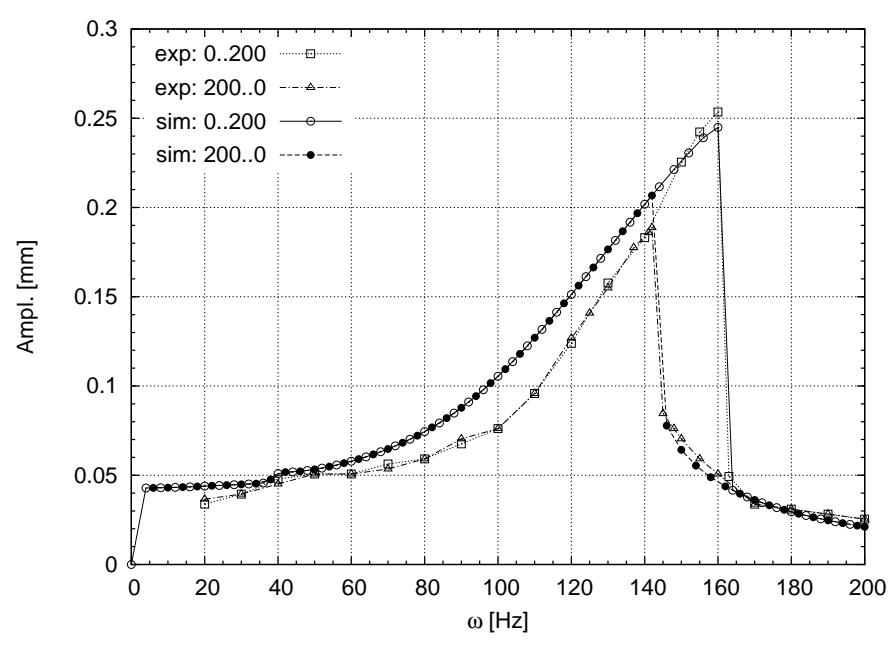

Fig. 8. Amplification diagrams obtained from experiment and simulation

\section{Conclusions}

We have given the mathematical model of an own-developed radial flux linear-motor with air-gap solenoid. We have also proved by numerical simulations that the analytical results based on Poincaré's method of small parameters give us acceptable information on the behaviour of the forced system and the comparison of amplification diagrams showed the correctness of the determined system parameters.

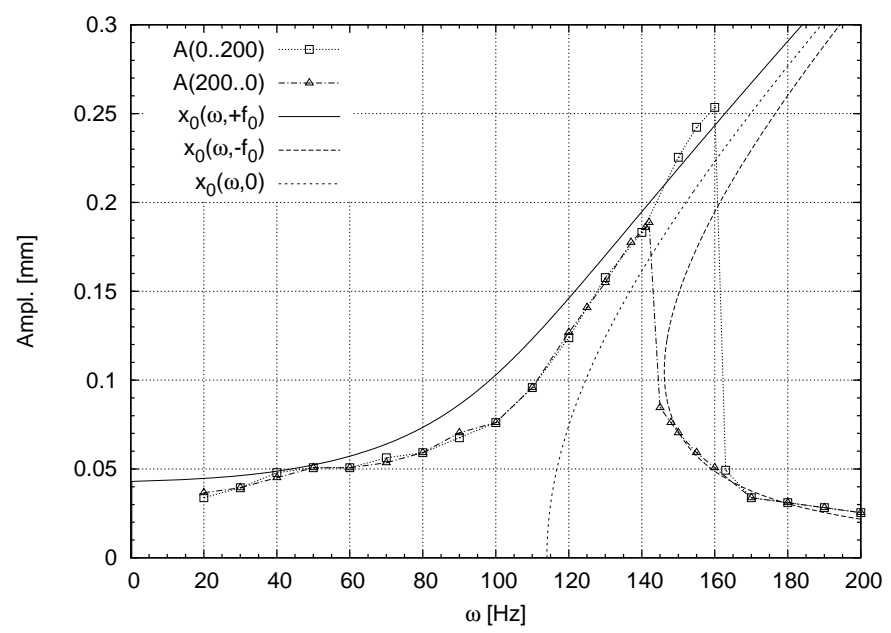

Fig. 9. Comparison of the measured diagram $(A(\omega))$ to the analytical curves $\left(x_{0}(\omega, \cdot)\right)$

\section{References}

1 Boldea I, Nasar S A, Linear Electric Actuator and Generator, University Press, Cambridge, 1997.

2 Chung S U, Hwang G Y, Hwang S M, Development of brushless and sensorless vibration motor used for mobile phone, IEEE Transactions on Magnetics 38 (2002), no. 5, 3000-3002.

3 Farkas M, Periodic Motions, Springer-Verlag, New York, 1994.

4 Köder T, Schinköthe W, Untersuchung zur Zuverlässigkeit von DC Kleinstmotoren, System-Zuverlässigkeit in frühen Entwicklungsphasen Workshop, September 27, 2004, pp. 1-12.

5 Linjama J, Kärkkäinen L, Modelling and characterization of vibrating alerts, Nordic Vibration Research, May 11, 2001.

6 Oesingmann D, Entwicklungsrichtungen dauermagneterregter Kommutatormotoren und bürstenlosen Motoren, 41. Internationales Wissenschaftliches Kolloquium, September 26, 1996, pp. 63-68. Band 2.

7 Wakiwaka H, Ezawa M, Kato H M, Yoshimura W, Development of highperformance millimeter-size vibrator for cellular phones, Journal of Magnetism and Magnetic Materials (2002), 242-245, 1195-1197. Part 2. 